\title{
Efecto de factores físico-sociales sobre la degradación del bosque nativo*
}

\author{
Effects of social and physical factors on native forest degradation \\ GERARDO VERGARA, JORGE GAYOSO \\ Instituto de Manejo Forestal, Universidad Austral de Chile, \\ Casilla 567, Valdivia, Chile.
}

\begin{abstract}
SUMMARY
The environmental impact on the native forests of the Province of Valdivia by access roads and populations was analyzed in this study. In agreement with other studies, the most significant impact on the forest occurred within a strip of $3 \mathrm{~km}$ on each side of the road, where the rate of deforestation was between $22 \%$ and $51 \%$. A model comprising physical and social factors explained the driving forces of forest degradation. Some biophysical variables and a high percentage of rural populations explained most of the intervention in the forest. A Principal Component Analysis proved an adequate technique for evaluating the importance of factors and their relationship with the spatial component.
\end{abstract}

Key words: roads, multivariate techniques, deforestation, GIS, environmental impact.

\section{RESUMEN}

Se analizó el impacto ambiental generado por los caminos y la influencia que ejercen los centros poblados sobre los bosques nativos de la provincia de Valdivia. En concordancia con otros estudios, se observó que el mayor impacto sobre el bosque se produce hasta una distancia de $3 \mathrm{~km}$ a cada lado del camino, llegando a ser deforestado -desde un punto de vista histórico- desde un $22 \%$ y hasta un $51 \%$ de la superficie original en ese espacio. Un modelo compuesto por factores sociales y físicos explica los cambios y degradación del bosque. Algunas variables biofísicas y un alto porcentaje de población rural son las variables que mejor explican la existencia de alteraciones en el bosque. El empleo de Análisis por Componentes Principales resultó una técnica adecuada para evaluar la importancia de las variables y su relación con la componente espacial.

Palabras claves: caminos, técnicas multivariadas, deforestación, SIG, impacto ambiental.

\section{INTRODUCCION}

La accesibilidad a los recursos es uno de los factores determinantes de cambio en la naturaleza; la existencia de nuevas vías de acceso como carreteras y caminos incrementan la presión hacia la utilización de los bosques. Por ello, determinar el impacto que producen los caminos de acceso so- bre los recursos forestales es de gran importancia para la planificación y protección de las zonas más vulnerables. Ello permite, además, evaluar o predecir el impacto de la construcción de nuevos caminos sobre los bosques.

Los caminos están estrechamente ligados a la presión que ejerce la población sobre el recurso para la extracción maderera y la utilización de éste

* Trabajo patrocinado por la Dirección de Investigación y Desarrollo de la Universidad Austral de Chile, DID S-1999053 con el título: "Metodología para estimar la fragilidad de ecosistemas forestales". 
BOSQUE 25(1): 43-52, 2004

Efecto de factores físico-sociales sobre la degradación del bosque nativo

como leña (1), y son un agente importante de cambio y degradación del bosque $(2,3)$. Uno de los casos más destacados relativos a los efectos ambientales producidos por la construcción de caminos corresponde a la Carretera Transamazónica en Brasil. Estos caminos fueron construidos con el propósito de proveer mejor acceso a regiones menos desarrolladas, pero a la vez tendieron a dar mayor valor a la propiedad para usos no forestales, fomentando la especulación de la tierra y la deforestación (3).

Muchos de estos procesos de cambio obedecen a la necesidad de obtener rápidamente terrenos para la agricultura o la ganadería. Como resultado de ello ocurrieron los grandes incendios de bosques durante el período de colonización en el sur de Chile, los que provocaron uno de los problemas ambientales más serios del país $(4,5,6)$

Además, otros factores tales como los bajos ingresos, la pobreza, el acceso restringido a la tecnología, la distancia a centros de consumo, el tipo de suelos, las características geomorfológicas y la propiedad de la tierra, hacen que zonas boscosas sean dedicadas a otros usos o actividades que se evalúan como más atractivas o rentables $(2,7,8)$

Apoyando estos argumentos, muchos autores han encontrado resultados donde muestran una alta correlación entre la presencia de caminos y altas tasas de deforestación; la mayor parte de estos estudios muestran que la tala de bosques declina rápidamente más allá de dos o tres kilómetros de distancia desde el camino, aunque otros reportan una tala de bosque importante hasta alrededor de $15 \mathrm{~km}$ desde los caminos más cercanos (7).

Otros aportes son entregados por Croissant (9), Nelson y Hellerstein (10) y Chomitz et al. (11), los cuales enfocan el análisis espacial a través de SIG y sensores remotos para determinar los efectos provocados por los caminos sobre la vegetación. Asimismo, Castillo et al. (12) estudian, con la ayuda de Sistemas de Información Geográficos, el efecto a escala espacial de los diferentes factores físicos y socioeconómicos que influyen en los cambios de uso del suelo y emisiones de gases invernadero.

Un completo análisis de los modelos de cambio de paisaje, su clasificación y las características más relevantes de éstos son realizadas por Baker (13). Por otro lado, Angelsen et al. (14) realizan una síntesis de los resultados de 150 modelos eco- nómicos de deforestación, describiendo sus supuestos, metodologías, datos y principales resultados, evaluando además sus fortalezas y debilidades.

El objetivo general de este trabajo es identificar y estimar el efecto de las variables o factores que determinan la degradación del bosque, basándose en las características biofísicas de la tierra, los indicadores sociales que caracterizan el sector y en los resultados de modelos utilizados en la literatura, con el propósito de entregar elementos que contribuyan a enfrentar el manejo forestal en forma sustentable y ser una herramienta de apoyo a la planificación territorial y las decisiones de política ambiental.

Este estudio se concentró en la provincia de Valdivia, la que se caracteriza por una intensa actividad agroganadera en la Depresión Intermedia, actividad tradicional en esta zona, y que, además, es caracterizada por poseer bosques de segundo crecimiento altamente fragmentados. El bosque nativo adulto se presenta en áreas de difícil acceso y de pendientes moderadas a fuertes de la Cordillera de los Andes y de la Costa. Asimismo, se desarrolla una creciente actividad forestal asociada a plantaciones de especies exóticas en sectores de pendientes medias a bajas de la Depresión Intermedia y la zona costera.

\section{MATERIAL Y METODOS}

El área de estudio comprende las doce comunas que componen la provincia de Valdivia, correspondientes a Corral, Futrono, Lanco, La Unión, Lago Ranco, Los Lagos, Máfil, Paillaco, Panguipulli, Río Bueno, San José de la Mariquina y Valdivia. La superficie total alcanza los $18.430 \mathrm{~km}^{2}$ y la población acumulada es de 330.000 habitantes aproximadamente (15). En conjunto forman una franja que abarca todo el territorio desde la zona costera hasta la Cordillera de los Andes, incorporando de esta manera los diversos factores que influencian el uso del suelo. La selección de las variables utilizadas se realizó basándose en la disponibilidad de información cartográfica proveniente de los organismos encargados de manejar recursos naturales (cuadro 1).

Para el análisis se utilizaron las descripciones de uso de suelo utilizadas por CONAF et al. (16), entendiéndose que éste más que un uso del suelo propiamente tal, corresponde muchas veces a una 


\section{CUADRO 1}

Variables incorporadas en el Análisis de Componentes Principales.

Variables included in the Principal Component Analysis.

\begin{tabular}{|c|c|c|}
\hline Variable & Descripción & Fuente \\
\hline $\begin{array}{l}\text { Distancia desde } \\
\text { Caminos }\end{array}$ & $\begin{array}{l}\text { Bandas a nivel comunal cada } 1.000 \mathrm{~m} \text { de distancia desde el borde } \\
\text { del camino, considerando los caminos públicos y privados de vialidad } \\
\text { mayor. }\end{array}$ & $(16)$ \\
\hline $\begin{array}{l}\text { Distancia desde } \\
\text { Centros Poblados }\end{array}$ & $\begin{array}{l}\text { Anillos concéntricos creados cada } 2 \mathrm{~km} \text { de distancia en torno a los } \\
\text { centros urbanos hasta una distancia de } 18 \mathrm{~km} \text {. Estos anillos fueron } \\
\text { generados sobre centros poblados con más de } 2.000 \text { habitantes. }\end{array}$ & $(15)$ \\
\hline $\begin{array}{l}\text { Densidad de } \\
\text { Caminos }\end{array}$ & $\begin{array}{l}\text { Cantidad lineal de caminos por unidad de superficie (comuna) ex- } \\
\text { presado en } \mathrm{m} / \mathrm{ha} \text {. }\end{array}$ & $(16)$ \\
\hline Uso del Suelo & $\begin{array}{l}\text { Información referente a la naturaleza y características de la vegeta- } \\
\text { ción, así como el uso que se da al suelo. }\end{array}$ & $(16)$ \\
\hline Pendiente & Rango medio de pendiente del sector analizado. & $(16)$ \\
\hline Altitud & Altura media sobre el nivel del mar de un área determinada. & $(16)$ \\
\hline Calidad del Suelo & $\begin{array}{l}\text { Definido a través de los procesos de formación u origen del suelo } \\
\text { (geomorfología). }\end{array}$ & $(17)$ \\
\hline Centros Poblacionales & $\begin{array}{l}\text { Considera el número de habitantes de la población urbana de la } \\
\text { comuna. }\end{array}$ & $(15)$ \\
\hline Ruralidad & $\begin{array}{l}\text { Indicador de la cantidad de personas que habitan en sectores rurales } \\
\text { con respecto a la población total. }\end{array}$ & $(15)$ \\
\hline Extrema Pobreza & $\begin{array}{l}\text { Indicador de la cantidad de personas que viven en extrema pobreza } \\
\text { respecto del total de personas de la comuna. }\end{array}$ & $(15)$ \\
\hline Ingreso Operacional & $\begin{array}{l}\text { Porcentaje de ingreso total de los municipios derivado de la activi- } \\
\text { dad propia de la entidad. }\end{array}$ & $(15)$ \\
\hline
\end{tabular}

condición ecológica derivada del medio ambiente natural.

Para el estudio se utilizó una imagen Landsat 5 TM de febrero del año 2000 sin cobertura nubosa. Para el tratamiento de esta imagen se utilizó el software Erdas Imagine ${ }^{\circledR}$ 8.4, el muestreo sobre las coberturas vectoriales se realizó con Arcinfo ${ }^{\circledR}$ 8.0 y el manejo y análisis final de estas coberturas se ejecutó con Arcview ${ }^{\circledR} 3.1$.

El análisis consideró la intersección de las coberturas de información y luego se realizó un muestreo sistemático cada $3 \mathrm{~km}$ de distancia a base de una cuadrícula sobrepuesta sobre la gráfica en la zona de estudio. Esto generó una muestra de 1.800 puntos, los cuales se georreferenciaron y cargaron con la información registrada en la base de datos de cada uno de estos puntos. Los datos de esta muestra fueron depurados con el fin de evitar la utilización de puntos que porten información no clasificada o que correspondan a zonas como cuerpos de agua o zonas sin cobertura aerofotogramétrica.

Todas las variables se estandarizaron a media 0 y varianza 1, para evitar que los límites de los 
intervalos de variación y los valores de cada índice en dicho intervalo perturben los resultados, debido a la magnitud que alcanzan los valores particulares de los índices basándose en sus intervalos de variación. Además, con el fin de simplificar la matriz de datos y evitar el "ruido" que provocan algunos tipos de uso de suelo, se excluyeron en la elaboración del índice los humedales y las categorías de terrenos sin vegetación, tales como suelos sobre el límite de vegetación, sectores rocosos o con nieve.

Posterior a la estandarización, las variables se analizaron por medio de Componentes Principales (ACP), para finalmente seguir los pasos propuestos por Moreno (18) para la interpretación estadística:

- Construcción de la matriz de datos.

- Revisión de la matriz de datos.

- Elección del número de componentes.

- Caracterización de los componentes o factores.

- Análisis de los gráficos de individuos y de las variables.

Influencia de los caminos y la población: Con el propósito de analizar el efecto de la accesibilidad en el uso de la tierra y estimar el grado de impacto que tiene en la degradación del bosque se realizó un análisis de distancias respecto a los caminos. Estas interrelaciones espaciales fueron analizadas a través de bandas alrededor de los caminos. El área con bosque dentro de cada banda fue dividida por el área total del banda, siendo posteriormente exportadas a Excel ${ }^{\circledR}$. En adición, se actualizó la información caminera con la ayuda de las imágenes digitales; sin embargo, cabe destacar que muchos caminos secundarios, en especial aquellos dedicados al madereo, no fueron detectados mediante este método.

El análisis se llevó a cabo en cuatro comunas de la provincia de Valdivia ubicadas en tres sectores caracterizados principalmente por sus atributos topográficos y geomorfológicos, cuya superficie conjunta es de $6.400 \mathrm{~km}^{2}$ aprox. El primer sector se concentra en las comunas de San José de la Mariquina y La Unión, que presentan la mayor parte de su superficie en la Cordillera de la Costa; un segundo sector lo compone la comuna de Paillaco, cuya superficie se concentra en la Depresión Intermedia. Finalmente, el tercer sector lo constituye la comuna de Futrono, cuyo territorio abarca principalmente la zona andina.

Por otro lado, se realizó un análisis de la relación de los centros poblados con respecto al uso del suelo con el propósito de analizar el impacto que la población genera sobre la vegetación circundante. Para este efecto, se generaron anillos concéntricos sobre cuatro centros poblados según cantidad de habitantes: Valdivia -capital provincial (120.000 hab. aprox.), San José de la Mariquina y Lago Ranco- capitales comunales (15.000 y 10.000 hab. respectivamente) y para el pequeño poblado de Malalhue, en la comuna de Lanco, con 2.000 habitantes. El radio máximo del anillo alrededor de los poblados se determinó a base del impacto directo visualizado en la imagen satelital sobre la vegetación arbórea.

\section{RESULTADOS Y DISCUSION}

\section{ANALISIS ESPACIAL PARA DETERMINAR EFECTOS SOBRE EL BOSQUE}

a) Influencia de los caminos. La figura 1 muestra el uso de la tierra, mientras las unidades territoriales se alejan de los caminos. Aquí se representan los cuatro principales usos del suelo, el porcentaje restante del área por banda es ocupado por otros tipos de uso tal como: bosques achaparrados, zonas urbanas e industriales, terrenos sin vegetación, entre otros. Los bosques achaparrados no se incluyeron en la gráfica debido a la condición natural donde éstos se desarrollan, sectores donde existe una baja presión de uso o las alternativas de producción son reducidas.

Se aprecia cómo el bosque nativo (bosque adulto y renoval) se conserva con niveles de cobertura sobre $80 \%$ en las unidades alejadas a más de 3.000 $\mathrm{m}$ de los caminos. Esto es coincidente con las afirmaciones de Angelsen y Kaimowitz (7) en cuanto a la distancia máxima de influencia de los caminos y su influencia sobre el bosque. A mayores distancias, el porcentaje restante del área de la banda es, por lo general, ocupado por bosques achaparrados o terrenos sin vegetación. Esto es muy claro en las comunas más forestales y de topografía más accidentada (Futrono y Corral), mientras en comunas que tradicionalmente han sido más agrícolas y de topografía más suave (Paillaco y San José) esto ocurre a los 4 ó $5 \mathrm{~km}$. 

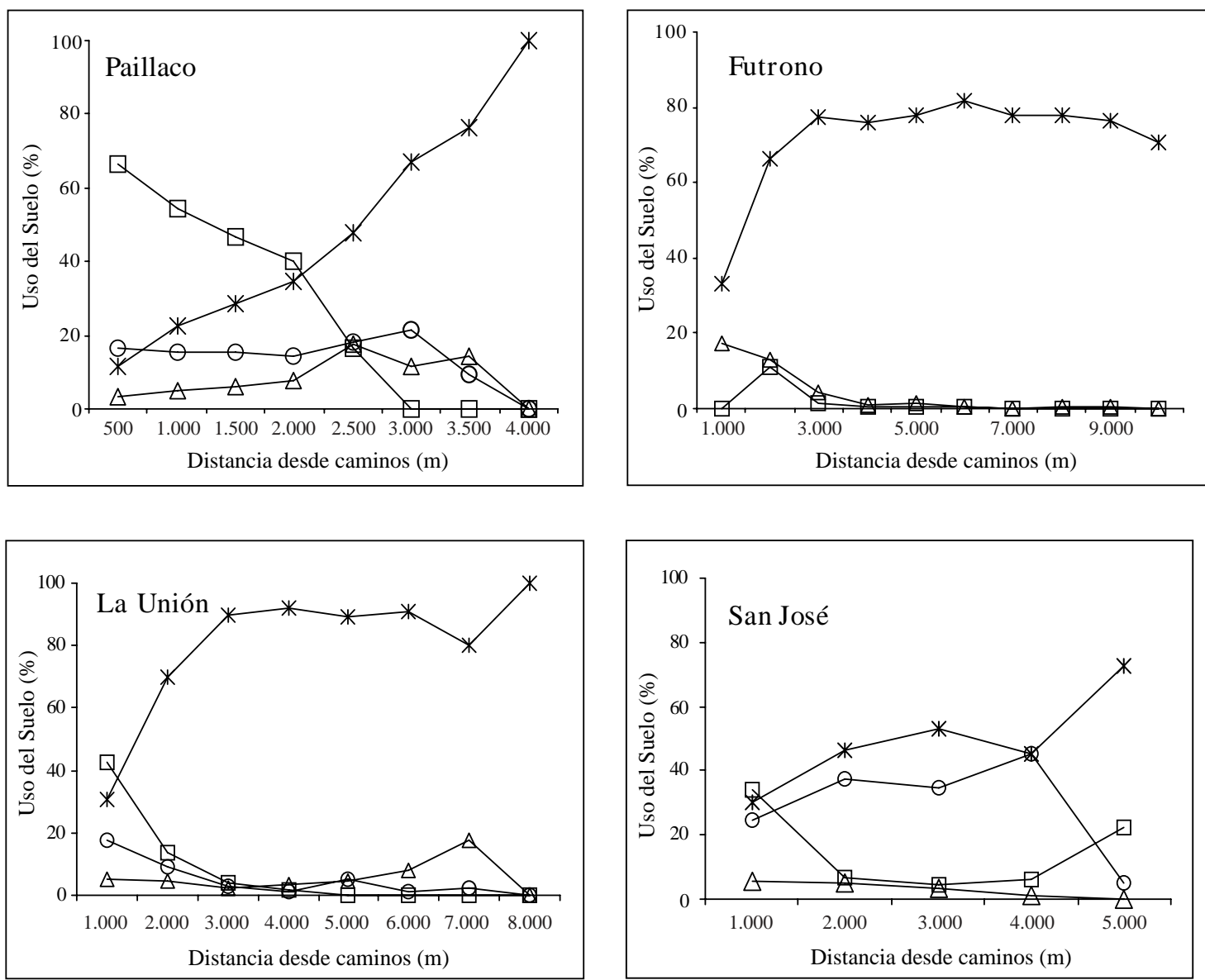

$\triangle \Delta$ Matorrales $\longrightarrow$ Plantación $\quad \square \quad$ Terrenos Agrícolas $\longrightarrow$ Bosque Nativo

Figura 1. Relación entre el uso del suelo y distancia desde caminos para cuatro comunas de la Provincia de Valdivia.

Relationships between land use and distance from roads in four counties of Valdivia Province.

En sentido contrario se aprecian más áreas dedicadas a la agricultura en las bandas más próximas a cada lado de los caminos y el efecto de competencia con el bosque es más evidente en la comuna de Paillaco (Depresión Intermedia). Además, esta comuna presenta una alta densidad de caminos concordante con una zona que tradicionalmente se ha dedicado a cultivos agrícolas y ganadería. Aquí existe un mayor porcentaje de renovales en zonas cercanas a caminos y mayores porcentajes de bosque nativo adulto a medida que nos alejamos de éstos. Cerca de un $60 \%$ de terreno es dedicado a la agricultura y praderas dentro de una distancia de $1.000 \mathrm{~m}$, desapareciendo totalmente sobre una distancia de $3.000 \mathrm{~m}$.
Las comunas de Paillaco y San José de la Mariquina concentran el mayor porcentaje de plantaciones con un $20 \%$ y $40 \%$ respectivamente; este porcentaje es importante hasta los $1.000 \mathrm{~m}$ en La Unión y es casi inexistente en Futrono. En las curvas de bosque nativo de las comunas de La Unión y Futrono, se aprecia una caída después de los $7.000 \mathrm{~m}$, lo que puede deberse a la interacción con otros caminos, o bien, a la presencia de caminos no registrados. En La Unión las actividades agrícolas y ganaderas se reducen fuertemente a los 3.000 m; en cambio, en San José de la Mariquina esta caída en la curva se produce a los $2.000 \mathrm{~m}$.

Una estabilización de la curva de bosque nativo se produce en promedio entre los 2.000 y 3.000 m, 
BOSQUE 25(1): 43-52, 2004

Efecto de factores físico-sociales sobre la degradación del bosque nativo

después de esta distancia este tipo de uso de suelo ocupa hasta un $80 \%$ de la superficie total de cada banda. El análisis de las superficies de las bandas de $1 \mathrm{~km}$ adyacentes a los caminos permite señalar que la deforestación promedio para el conjunto de comunas estudiadas alcanza al $51 \%$ de la superficie de estas bandas; esto es, aproximadamente 102 $\mathrm{ha} / \mathrm{km}$. La segunda banda posee un menor grado promedio de intervención del bosque que bordea el $26 \%$ del total de ésta. Vistos en su conjunto, los caminos han afectado en promedio el $22 \%$ de la superficie territorial neta de las comunas.

Sin embargo, cabe destacar que este análisis es realizado desde el punto de vista histórico, ya que en este caso la curva de bosque nativo incluye la categoría de bosques secundarios o de segundo crecimiento, lo que enmascara de alguna manera el impacto original de los caminos de acceso más antiguos.

El análisis de las superficies sugiere, por tanto, que la intervención del bosque nativo ha sido influenciada marcadamente por el nivel de acceso que define la distancia de madereo y los costos de producción de la madera. Además, se aprecia que el ancho de intervención de bosques es menor en las comunas de topografías accidentadas en relación a las más planas, asociándose también un mayor costo de producción a las mayores pendientes.

Sin duda, un factor importante en la variación de la distribución de bosques y praderas es la pendiente del terreno, la cual mitiga el efecto de la presión sobre los bosques, debido a que los terrenos escarpados no son adecuados para la agricultura y, por otro, el costo de extracción de la madera es más elevado. La figura 2 muestra el efecto de las pendientes sobre el uso del suelo, las cuales determinan que para sectores de pendientes moderadas o bajas se produzca una mayor presión hacia la utilización agrícola-ganadera, quedando el bosque restringido a sectores de difícil acceso y topografía más accidentada. La reducción del porcentaje de bosque nativo sobre $60 \%$ de pendiente se debe en parte a las condiciones restrictivas de suelo y arraigamiento que enfrentan los árboles, siendo ocupados generalmente por bosques achaparrados o terrenos descubiertos de vegetación, los cuales no se incluyeron en la gráfica.

b) Influencia de la población. La presión que ejerce la población sobre los bosques es más marcada que la ocasionada directamente por los caminos;

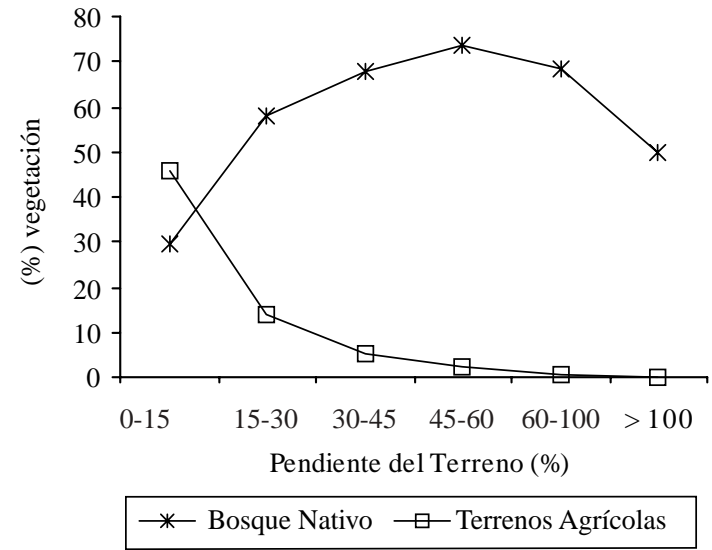

Figura 2. Relación entre pendiente del terreno y deforestación.

Relationship between ground slopes and deforestation.

no obstante, sigue similar tendencia. Los cultivos agrícolas y praderas siempre ocupan el primer lugar a menores distancias de los poblados, independiente del tamaño y poblado elegido. En contraposición a lo esperado, ciudades de mayor tamaño poblacional, como Valdivia, no se diferencian mayormente de los poblados más pequeños, presentándose en general un incremento de la presencia de bosque nativo entre los 8.000 y 10.000 m (figura 3).

En este caso la influencia ejercida por la población es fuertemente enmascarada por los factores geomorfológicos, como es el caso de la Cordillera de la Costa en Valdivia y de los Andes en Lago Ranco. Por otro lado, San José de la Mariquina y Malalhue presentan menores porcentajes de bosque nativo a lo esperado debido a las aptitudes eminentemente agrícolas de los terrenos circundantes. Los factores topográficos, como la pendiente, tienen un efecto importante sobre la utilización del suelo alrededor de los poblados, ya que la mayoría de las ciudades se ubica en sectores planos o de bajas pendientes, lo que hace que los terrenos cercanos se dediquen a actividades agropecuarias cuando éstas son más rentables (8).

Modelación del cambio: Esta etapa realizada a través de ACP se llevó a cabo con el propósito de identificar las variables más influyentes en la degradación o pérdida de bosque nativo. A partir de los datos estandarizados, se obtuvo la matriz de correlaciones entre las variables en estudio (cuadro 2). La variable que muestra la más alta co- 

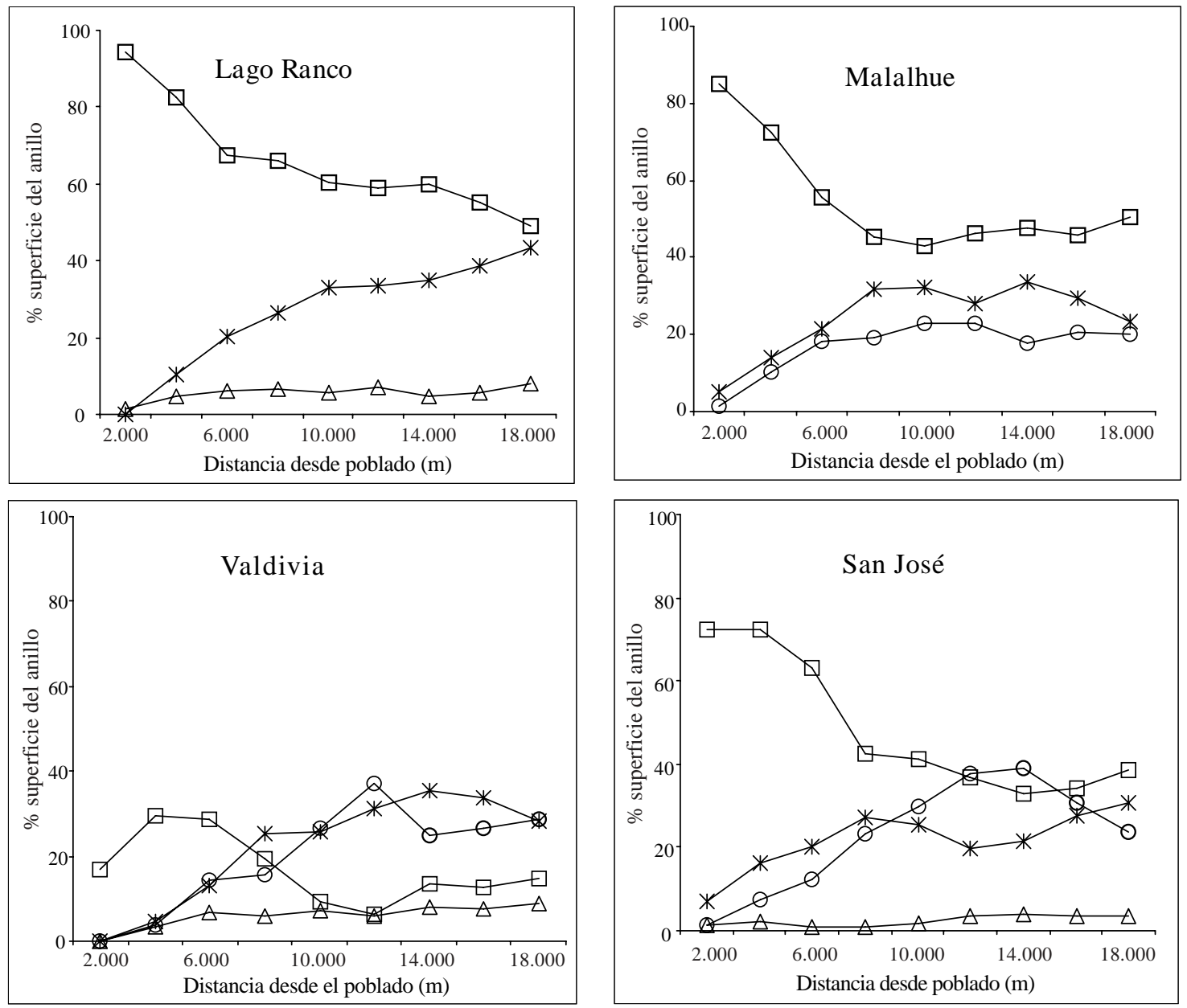

$\triangle$ Matorrales $\longrightarrow$ - Plantación $\square \square$ Terrenos Agrícolas $\longrightarrow$ Bosque Nativo

Figura 3. Relación entre el uso del suelo y la distancia desde los poblados.

Relationships between land use and distance from towns.

rrelación con el uso del suelo corresponde a Altitud con un $75 \%$, seguida de las variables Distancia desde Caminos, Pendiente, Suelos y Distancia desde centros poblados.

El gráfico de Cattell, representado en la figura 4, muestra que el primer componente explica el $45,17 \%$ de la variación y los tres primeros componentes suman un $65,53 \%$. Los coeficientes del primer vector propio (cuadro 3 ) indican que esta combinación lineal es aproximadamente un promedio entre todas las variables, con ponderación positiva para las primeras ocho variables y valores negativos para Ingreso Operacional, Número de Habitantes y Densidad de Caminos, lo que indica que disminuirá el valor del primer componente si aumentan los valores de las variables con ponde- ración negativa. En este caso, el primer vector está representado por variables físicas y de carácter social como Ruralidad y Extrema Pobreza, las cuales determinan el acceso y nivel de impacto sobre el bosque.

El segundo vector propio muestra valores semejantes para Densidad de Caminos y las primeras seis variables, con coeficientes negativos para las variables Ruralidad y Extrema Pobreza, lo que reducirá el valor de este componente cuando aumenten el valor estas últimas. Las variables Ingreso Operacional y Centros Poblacionales muestran los más altos coeficientes positivos; de esta manera los sectores con valores más elevados del segundo componente principal serán aquellos que posean mayores valores de estas variables, consti- 
BOSQUE 25(1): 43-52, 2004

Efecto de factores físico-sociales sobre la degradación del bosque nativo

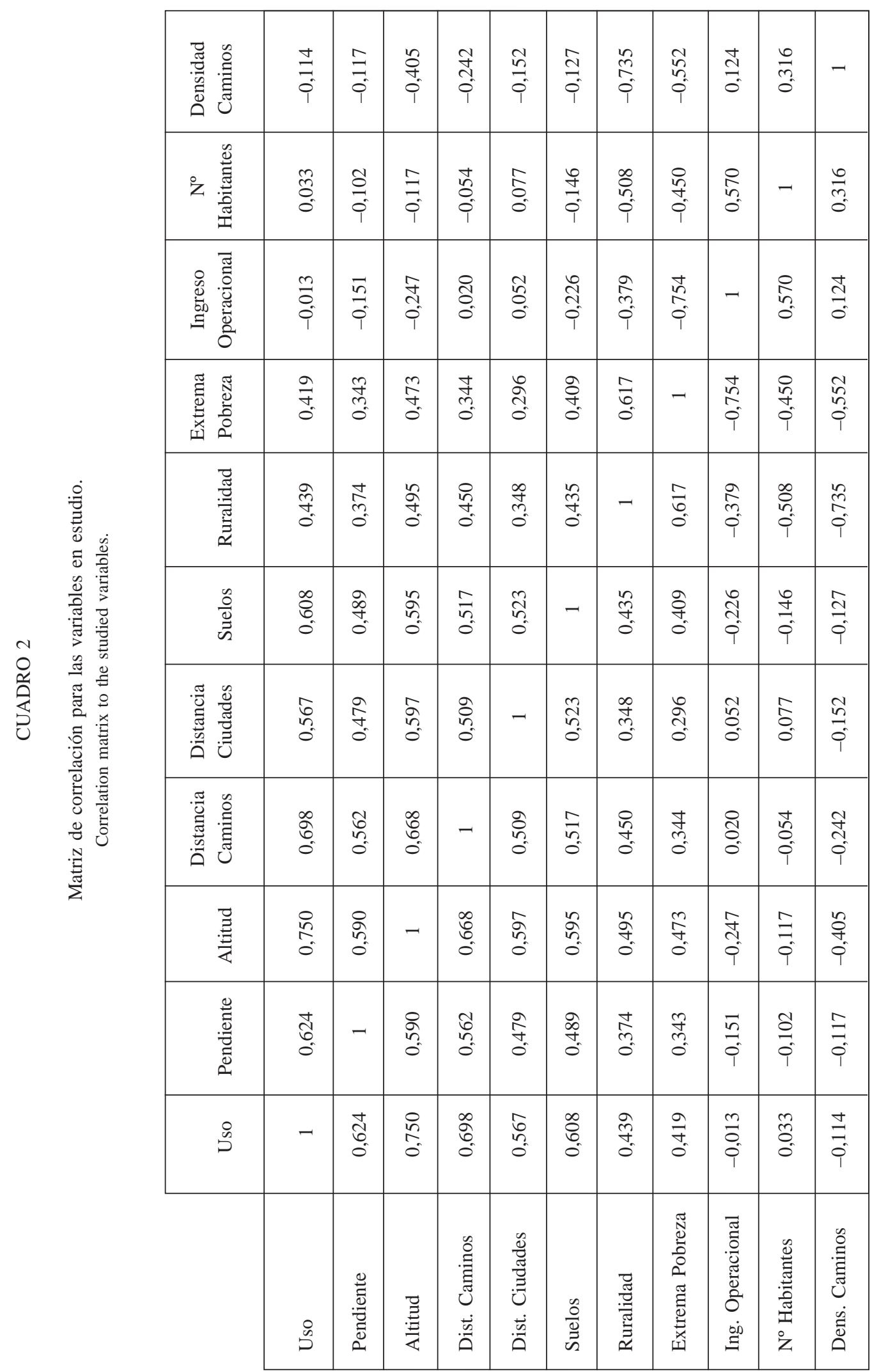




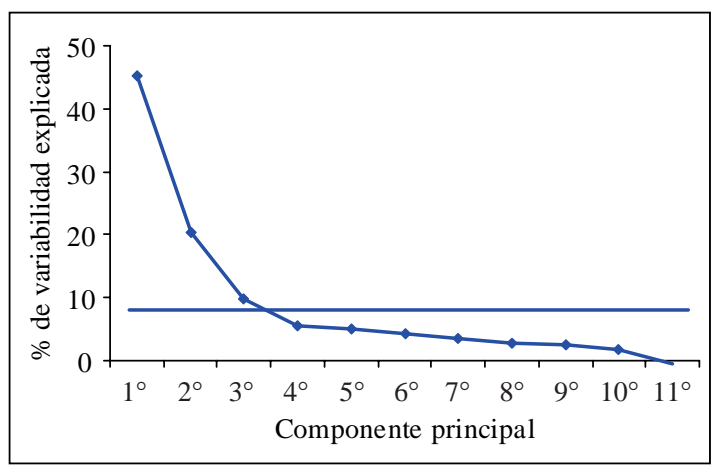

Figura 4. Gráfico de Cattell mostrando la variación explicada por cada componente.

Cattell diagram showing the explained variation of every component.

tuyendo un claro ejemplo del efecto de las variables de tipo económico. Un caso particular lo constituye el tercer vector propio, donde el coeficiente más alto lo ocupa la variable Densidad de Caminos con valores negativos y positivos, pero de menor ponderación para las otras variables.

A partir de los resultados, se destaca el fuerte efecto que poseen las variables físicas sobre la deforestación; sin embargo, queda demostrado, a través de este análisis, que las variables socioeconómicas son importantes en la explicación de cambios de uso del suelo, claro está, que con un menor grado de correlación que el primer grupo de variables, debido tal vez a la falta de desagregación y al difícil tratamiento de estas variables en un análisis de tipo espacial.

\section{CONCLUSIONES}

El impacto que generan las actividades antrópicas sobre el bosque depende de una compleja interrelación de variables y que responde a diversas razones, entre ellas las político-económicas. Sin duda, uno de los mayores problemas es poder contar con la información adecuada para realizar estimaciones más precisas y enfocadas a la realidad del medio. En muchos casos uno de los grandes problemas encontrados fue dar con variables sociales lo suficientemente desagregadas espacialmente y contar con la información adecuada acerca de las características de la tierra, ya que, en muchos casos, no existe en su dimensión espacial o poseen elevados costos para incluirlas en un estudio.

Se confirma que los caminos son un agente que promueve la deforestación agregando mayor tensión al uso de los recursos naturales. Al igual que en otros estudios, se comprueba que existe un

\section{CUADRO 3}

Correlaciones entre variables iniciales y los componentes principales. Correlations between the original variables and the principal components.

\begin{tabular}{|l|r|r|r|r|r|}
\cline { 2 - 6 } \multicolumn{1}{c|}{} & Factor 1 & Factor 2 & Factor 3 & Factor 4 & Factor 5 \\
\hline Uso & 0,7806 & 0,4295 & 0,0853 & $-0,0303$ & $-0,1372$ \\
Pendiente & 0,6968 & 0,2815 & 0,2159 & $-0,3013$ & $-0,2464$ \\
Altitud & 0,8520 & 0,2011 & $-0,0199$ & 0,0938 & $-0,1262$ \\
Dist. Caminos & 0,7417 & 0,3580 & $-0,1021$ & $-0,2485$ & $-0,1357$ \\
Dist. Ciudades & 0,6448 & 0,4299 & $-0,0423$ & 0,2908 & 0,3338 \\
Suelos & 0,7265 & 0,1956 & 0,2633 & 0,0473 & 0,4229 \\
Ruralidad & 0,7682 & $-0,3743$ & $-0,3423$ & $-0,1146$ & 0,1260 \\
Extrema pobreza & 0,7346 & $-0,4986$ & 0,1215 & 0,3097 & $-0,1843$ \\
Ing. Operacional & $-0,3892$ & 0,7021 & $-0,5269$ & $-0,1903$ & 0,1234 \\
No Habitantes & $-0,3391$ & 0,7233 & $-0,1249$ & 0,4488 & $-0,2558$ \\
Dens. Caminos & $-0,5076$ & 0,4241 & 0,7135 & $-0,1080$ & 0,0700 \\
\hline
\end{tabular}


BOSQUE 25(1): 43-52, 2004

Efecto de factores físico-sociales sobre la degradación del bosque nativo

impacto directo a través de las vías de acceso sobre el bosque hasta una distancia de 2.000 ó 3.000 $\mathrm{m}$, dependiendo de las características de la comuna, llegando en promedio a deforestarse hasta un $50 \%$ del bosque nativo a una distancia de $1 \mathrm{~km}$ desde el camino. Por otro lado, la influencia de los centros poblados sobre el bosque es más marcada, producto de la utilización de estos suelos a actividades agrícolas e industriales.

Respecto al análisis de variables, aquellas de carácter físico son las que muestran una asociación directa con el uso del suelo, seguidas por aquellas de tipo económico-social. El análisis por Componentes Principales permitió realizar un eficiente análisis espacial de las variables estudiadas y de su influencia sobre la existencia o degradación del bosque. Es, por lo tanto, imprescindible para futuros estudios contar con información desagregada que pueda mostrar con mayor claridad los fenómenos de cambio de uso en el suelo.

\section{BIBLIOGRAFIA}

(1) DRIGO, R. Population dynamics and the assessment of land use changes and deforestation, part 1. 1999. [citado 14 Junio de 2000]. Disponible en: <http://www.fao .org/ sd/ WPdirect/Wpan0030.htm>.

(2) ROPER, J., R. ROBERTS. Deforestation: Tropical Forest in Decline. Canadá. 1999. [citado 14 Junio de 2000]. Disponible en: <http://www.rcfa-cfan.org/English/issues 12.html>

(3) VITERI, M. A. Diagnóstico de la fragilidad de ecosistemas de bosque nativo con criterio ambiental. Chile Forestal, $1998, \mathrm{~N}^{\circ} 258$, p. $20-21$

(4) HARTWIG, F. Chile. Desarrollo Forestal Sustentable. Ensayo de política forestal. Santiago, Chile. Ed. Los Andes. 1991. $195 \mathrm{p}$.

(5) CODEFF. El futuro del bosque nativo chileno: un desafio de hoy. Santiago, Chile. 1992. 54 p.

(6) CATAlAN, R., R. ANTIQUEO. Pueblo mapuche, bosque nativo y plantaciones forestales. Las causas subya- centes de la deforestación en el sur de Chile. Ediciones Univ. Católica de Temuco, Chile. 1999. 81 p.

(7) ANGELSEN, A., D. KAIMOWITZ. Rethinking the causes of deforestation: Lessons from economic models. In The World Bank Research Observer. 1999 [citado 14 Junio de 2000]. Disponible en: 〈http://www.worldbank.org>.

(8) CROOPER, M., C. GRIFFITHS, M. MANI. Roads, population pressures, and deforestation in Thailand, 1976-89. The World Bank. Washington D.C. 1997. [citado 14 Junio de 2000]. Disponible en: <http://www. worldbank. org>

(9) CROISSANT, C. Roads, towns and forest: the use of GIS and remote sensing in determining the effect of proximity to roads and settlements on the location of forest near Sotuta, Mexico. 1998. [citado 14 Junio de 2000]. Disponible en: 〈http://www .indiana.edu/ cipec/>.

(10) NELSON, G., D. HELLERSTEIN. Do roads cause deforestation? Using satellite images in econometric analysis of land use. Amer. J. Agr. Econ., 1997, Vol. 79, p. $80-88$.

(11) CHOMITZ, K., D. GRAY. Roads, land use, and deforestation: A spatial model of land use in Belize. The World Bank policy research working paper 1444, Washington D.C. 1995. [citado 14 Junio de 2000]. Disponible en: <http://www.worldbank.org>.

(12) CASTILLO, M., G. HELLIER, B. DE JONG, R. TIPPER Estimación de una línea de base regional, un enfoque geográfico. IUFRO-RIFALC. Taller internacional sobre secuestro de carbono. 16 y 17 julio de 2001. Mérida, Venezuela. 2001. 9 p.

(13) BAKER, W. L. A review of models of landscape change. Landscape Ecology. Vol. 2, No 2, p. 111-1133, 1989.

(14) KAIMOWITZ, D., A. ANGELSEN. Economic models of tropical deforestation. A review. Center for International Forestry Research, Malaysia. 1998. 134 p.

(15) INSTITUTO NACIONAL DE ESTADISTICAS. Chile: División político-administrativa. Subdirección Técnica; Santiago, Chile. 1995. 139 p.

(16) CONAF, CONAMA, BIRF. Catastro y evaluación de recursos vegetacionales nativos de Chile. Informe Décima Región. Univ. Austral de Chile, Pontif. Univ. Católica de Chile, Univ. Católica de Temuco. 1999

(17) SCHLATTER, J. Principales grupos de suelos según material de origen. Facultad de Cs. Forestales. Univ. Austral de Chile. Nota técnica. 1998.

(18) MORENO, R. Propuesta metodológica para la asignación de subsidios federales orientados a la atención de la población marginada. Tesis Magíster Economía y Gestión Regional. Valdivia, Chile. Universidad Austral de Chile, Fac. Cs. Económicas. 2000. 138 p. 